\title{
Epidemiological Characteristics of Fournier's Gangrene: A Report of 71 Patients
}

\author{
I. Atilla Aridogan Volkan Izol Deniz Abat Onur Karsli Yildirim Bayazit \\ Nihat Satar \\ Department of Urology, Faculty of Medicine, University of Çukurova, Adana, Turkey
}

\section{Key Words}

Necrotizing fasciitis • Fournier's gangrene $\cdot$ Predisposing

factors $\cdot$ Epidemiology

\begin{abstract}
Introduction: To identify the predisposing factors, etiological and clinical characteristics as well as the Fournier's gangrene (FG) severity index (FGSI) in the outcomes of patients with FG. Materials and Methods: The data from 71 patients diagnosed with FG in a period of 17 years were retrospectively reviewed for the age of the patient, their history, predisposing factors, etiology, prodromal symptoms, FGSI, culture results, hospitalization period, surgical interventions, responses to the therapy and complications. Results: All of the patients were Caucasian males, and the mean age was 61.3 (range 36-92) years. The mean duration from the onset of symptoms to surgery was 7.5 days. The mean hospitalization time was 27.4 days and the most common etiological factor for FG was a perineal abscess. The overall mortality rate was $29.6 \%$. The relationship between the number of predisposing factors and mortality rates in patients with FG was the most statistically significant parameter $(p=0.001)$. Conclusions: Multiple predisposing factors predict a poor prognosis and correlate significantly with mortality. Exten-
\end{abstract}

sion of the disease and the FGSI score were not predictive of outcome. The most essential intervention for stopping the rapidly progressing infectious process of FG consists of early recognition of the disease, proper management of the predisposing factors and aggressive surgical debridement. Such an intervention can improve clinical outcomes.

Copyright $\odot 2012$ S. Karger AG, Basel

\section{Introduction}

Fournier's gangrene (FG), a rapidly progressive inflammation and necrosis of the subcutaneous tissue and fascia, was first described by Dr. Jean Alfred Fournier in 1877 [1]. He described the disease in a specific region of the body and used the term fulminant gangrene of penis and scrotum. His description was based on 3 major findings: (1) the abrupt onset in a healthy young male patient, (2) a rapid progression to gangrene and (3) an unknown etiology. Recently, FG has been termed necrotizing fasciitis of the perirectal, perianal and genital areas as the result of subcutaneous vessel thrombosis with obliterative endarteritis and gangrene overlying the dermal and epidermal tissues due to mostly both polymicrobial aerobic and anaerobic bacteria [2-4].

\section{KARGER}

Fax +4161306 1234

E-Mail karger@karger.ch

www.karger.com (c) 2012 S. Karger AG, Basel

0042-1138/12/0894-0457\$38.00/0

Accessible online at:

www.karger.com/uin
Volkan Izol, MD

Department of Urology, Faculty of Medicine

University of Çukurova

TR-01330 Sariçam, Adana (Turkey)

E-Mailvolkanizol@yahoo.com 
Table 1. Etiological factors of FG in 71 patients

\begin{tabular}{lrr}
\hline Etiology & \multicolumn{2}{l}{ Patients } \\
\cline { 2 - 3 } & $\mathrm{n}$ & $\%$ \\
\hline Perineal abscess & 22 & 31 \\
Chronic urethral catheterization & 14 & 20 \\
Bladder cancer & 9 & 13 \\
Following traffic accident & 5 & 7 \\
Posttraumatic bladder and urethra injury & 5 & 7 \\
Rectum cancer & 5 & 7 \\
Suprapubic catheter insertion & 5 & 7 \\
Genital shaving & 3 & 4 \\
Scrotal irreducible hernia & 2 & 3 \\
Prostatic abscess & 1 & 1 \\
\hline
\end{tabular}

FG generally affects elderly people of both sexes and different risk factors have been implicated including diabetes mellitus, rectal and perianal abscesses, hypertension, local trauma, urethral stricture, chronic catheterization and chronic renal failure [5-7]. It is important to diagnose and treat FG rapidly because of its high mortality (range 7-75\%) and morbidity rates [7]. Early surgical intervention/debridement with the use of broad-spectrum antimicrobial agents and intensive care techniques are essential for FG treatment in order to reduce morbidity and mortality. In this study, we retrospectively evaluated the predisposing factors, the etiological, clinical and microbiological characteristics as well as the FGSI (FG severity index) [1] in the outcomes of 71 FG patients.

\section{Patients and Methods}

We analyzed the data from 71 patients who were diagnosed with FG and managed in our department over a period of 17 years. The records were retrospectively reviewed for patients' age, medical history, predisposing factors, etiology, prodromal symptoms, FGSI, culture results, hospitalization period, surgical interventions, responses to the therapy and complications. The clinical findings upon physical examination are important for the diagnosis of FG, i.e. erythema, rash, swelling, tenderness, fluctuation, subcutaneous emphysema and necrosis. The body surface area nomograms were used for calculating the extent of the disease. The penis, scrotum and perineum each accounted for $1 \%$ and each ischiorectal fossa for $2.5 \%$ as described [1]. Aggressive surgical debridement was performed under general anesthesia in all patients until normal fascia was found, and subsequent repeated daily debridements were performed at bedside. Suprapubic diversion and/or colostomies were performed in cases that had suspected urinary extravasation and/or fecal contamination. The intravenous antibiotic combination therapy included a third-gener- ation cephalosporin, an aminoglycoside and metronidazole. This therapy was initiated empirically until obtaining the results of microbiological analysis, at which point it was continued or changed according to the results of culture and sensitivity. The patients were evaluated 6 weeks after their discharge and this is accepted as the time of survival.

Statistical Analysis

A Mann-Whitney test was used to compare the distribution of albumin, alkaline phosphatase (ALP), FGSI scores and age values among survivors and nonsurvivors. For categorical data, a $\chi^{2}$ test was used to compare the mortality rates defined by predisposing risk factors.

\section{Results}

All of the patients were Caucasian males, and ages ranged from 36 to 92 years, with an average of 61.3 years. The distribution of cases shows uniformity over the years. Each year, 3-5 patients were treated. Of the 71 patients reviewed, 21 (29.6\%) died and 50 (70.4\%) survived. The mean ages of survivors and nonsurvivors were $61.2 \pm$ 12.1 and $66 \pm 12.4$ years, respectively. The difference in ages was found statistically not significant $(\mathrm{p}=0.48)$.

The mean duration from the onset of symptoms to surgery in survivors and nonsurvivors was $7.5 \pm 4.1$ and 7.5 \pm 3.6 days, respectively, and there was no statistically significant difference $(\mathrm{p}=0.12)$. At presentation, major prodromal symptoms included local pain and hyperemia, and physical exam findings included penoscrotal pain, edema, gangrene and a 'dead fetid odor'. Sepsis was observed upon admission in 2 and 12 of the surviving and nonsurviving patients, respectively $(p=0.001)$. All of the patients were operated on within $10 \mathrm{~h}$ of admission. The mean hospitalization time was 27.4 days (range: $3-56$ days) and was similar for survivors and nonsurvivors $(\mathrm{p}=0.60)$.

Perineal abscess was the most common etiological factor for FG (table 1). The most frequently observed predisposing factors included diabetes mellitus (50\%), hypertension (20\%), chronic renal failure (15\%) and/or congestive heart failure (11\%). Of 69 patients for whom information was complete, 29 had only one predisposing factor, 20 had two predisposing factors and 13 had three or more predisposing factors; the remaining 7 patients had no predisposing factors. However, no information had been provided on two patients discharged from the intensive care unit by their own choice. The mortality rate for patients with one, two or three predisposing factors was 17.2, 40 and $53.8 \%$, respectively. The relationship between the number of predisposing factors and mortality rates in patients with FG was statistically significant $(\mathrm{p}=0.001)$ (table 2$)$. 
Tissue and/or urine samples were obtained for microbiological analysis. A single microorganism was isolated in $14(19.7 \%)$ and polymicrobial infection was encountered in $57(80.3 \%)$ patients. The most frequently isolated microorganisms were as follows. Escherichia coli (36\%), Staphylococcus aureus (32\%), Candida albicans (18\%), Acinetobacter baumannii (10\%) and the rest of the microorganisms (24\%) are presented in table 3. In addition, anaerobic microorganisms were not recovered in our patients who had positive aerobic wound cultures.

The mean extent of the involved body surface area in survivors and nonsurvivors was $2.9 \pm 1.9$ and $3.7 \pm 2.1 \%$, respectively, and the difference was not statistically significant $(p=0.17)$. Aggressive surgical excision of necrotic tissue was performed and additional debridements under general anesthesia in the operating room were required for 21 patients, all of them survivors. A colostomy due to perirectal debridement was performed in 23 patients, 5 of which were nonsurvivors ( $p>0.05$ ), and all colostomies were closed at an average of 5 months (range 4-8 months) with the exception of 1 patient who had colon cancer. A unilateral orchiectomy due to necrosis of the testis was performed in 6 patients, and a bilateral orchiectomy was performed in 5. Testosterone replacement therapy was also planned for the patients who underwent bilateral orchiectomy. A suprapubic catheter was placed in 5 patients because of urethral debridement, and urinary drainage was maintained with a Foley urethral catheter for all other patients. During the postoperative period, daily hydrotherapy and superficial debridement was instituted if necessary. In 48 surviving patients, reconstruction was performed using myocutaneous flaps or dermal grafts after the wounds healed in 41 (85.4\%), and secondary wound healing was observed in the remaining 7 (14.6\%).

We obtained information about all nine parameters in 32 of 71 patients, and the mean FGSI scores for survivors and nonsurvivors were $5.7 \pm 2.3$ and $8.7 \pm 4.9$, respectively. The difference in FGSI scores were found not significant $(\mathrm{p}=0.08)$. The mean serum albumin levels of survivors and nonsurvivors were $2.61 \pm 0.97$ and $2.27 \pm$ 0.86 , respectively $(\mathrm{p}=0.45)$. In addition, ALP levels of survivors and nonsurvivors were $390 \pm 226$ and $991 \pm$ 1,349 , respectively $(\mathrm{p}=0.46)$. Analysis of age, time to consult, affected skin surface, hospitalization time, sepsis on admission and FGSI between survivors and nonsurvivors is shown in table 4.

Major complications included sepsis $(\mathrm{n}=9)$, urethrocutaneous fistula $(\mathrm{n}=2)$ and pneumonia $(\mathrm{n}=1)$. All of the nonsurvivors had sepsis, which was the most frequent reason for mortality.

Epidemiology of Fournier's Gangrene
Table 2. Relationship between the number of predisposing factors and mortality rates in 69 patients with FG

\begin{tabular}{lclll}
\hline $\begin{array}{l}\text { Number of } \\
\text { predispos- } \\
\text { ing factors }\end{array}$ & $\begin{array}{l}\text { Survivors } \\
(\mathrm{n}=48)\end{array}$ & $\begin{array}{l}\text { Non- } \\
\text { survivors } \\
(\mathrm{n}=21)\end{array}$ & $\begin{array}{l}\text { Total } \\
(\mathrm{n}=69)\end{array}$ & $\begin{array}{l}\text { Mortality } \\
\text { rate } \\
(\mathrm{NS} / \text { total })\end{array}$ \\
\hline 0 & $6(12.5 \%)$ & $1(4.8 \%)$ & $7(10.1 \%)$ & $14 \%$ \\
1 & $24(50 \%)$ & $5(23.8 \%)$ & $29(42 \%)$ & $17.2 \%$ \\
2 & $12(25 \%)$ & $8(38.1 \%)$ & $20(29 \%)$ & $40 \%$ \\
$\geq 3$ & $6(12.5 \%)$ & $7(33.3 \%)$ & $13(18.9 \%)$ & $53.8 \%$ \\
\hline
\end{tabular}

NS $=$ Nonsurvivors

Table 3. Etiological agents in 71 patients with FG

\begin{tabular}{lc}
\hline & Patients, $\mathrm{n}(\%)$ \\
\hline Escherichia coli & $26(36)$ \\
Staphylococcus aureus & $23(32)$ \\
Candida albicans & $13(18)$ \\
Acinetobacter baumannii & $7(10)$ \\
Gram-negative bacteria & $5(7)$ \\
Staphylococcus hominis & $4(6)$ \\
Staphylococcus warneri & $2(3)$ \\
Enterobacter cloacae & $2(3)$ \\
Klebsiella spp. & $2(3)$ \\
Pseudomonas aeruginosa & $1(2)$ \\
\hline
\end{tabular}

Table 4. Analysis of age, time to consult, affected skin surface, hospitalization time, sepsis on admission and FGSI between survivors and nonsurvivors

$\begin{array}{lll}\begin{array}{l}\text { Survivors } \\ (\mathrm{n}=50)\end{array} & \begin{array}{l}\text { Non- } \\ \text { survivors } \\ (\mathrm{n}=21)\end{array} & \mathrm{p} \\ & \text { value }\end{array}$

Age, years

Time to consult, days

Affected skin surface, $\%$

Mean hospitalization time, days

Sepsis on admission, $\mathrm{n}$

FGSI

Albumin, g/dl

Alkaline phosphates, U/l
$61.2 \pm 12.06 \quad 66.2 \pm 12.4 \quad 0.48$

$7.5 \pm 4.15 \quad 7.5 \pm 3.68 \quad 0.12$

$2.91 \pm 1.94 \quad 3.71 \pm 2.17 \quad 0.17$

$27.4 \pm 13.9419 .9 \pm 11.790 .30$

$\begin{array}{lll}2 & 12 & 0.001\end{array}$

$\begin{array}{lll}5.78 \pm 2.3 & 8.78 \pm 4.99 & 0.08\end{array}$

$\begin{array}{lll}2.61 \pm 0.97 & 2.27 \pm 0.86 & 0.45\end{array}$

$390 \pm 226 \quad 991 \pm 1,3490.46$

\section{Discussion}

FG is a life-threatening urological emergency, which is characterized by necrotizing fasciitis of the external genitalia and/or perineum. It is a rapidly progressive condition and an accurate diagnosis, early aggressive surgical 
treatment and intensive supportive care with broad-spectrum intravenous antibiotics are important steps in the management of FG and crucial for prognosis due to high morbidity and mortality rates (range 7-75\%) [1, 5, 7-10]. In our study, the mortality rate was $29.6 \%$ similar to some previous reports. In addition, predisposing factors are very important for FG prognosis because they are related to morbidity and mortality [7-9]. In this study, 71 male patients were treated over a period of 17 years for FG, and, despite treatment, most of the deaths were in patients with multiple predisposing factors. This study does have limitations e.g. its retrospective character, and because it goes back over a long time some of the data are missing. In addition, all of the patients were treated surgically and no other treatment modality such as hyperbaric oxygen therapy (HBO) was used.

The mean age of patients with FG has progressively increased in the published series. In our study, the mean age of survivors and nonsurvivors was similar, and there was no significant difference between the groups, as observed in prior studies [2, 11]. However, Laor et al. [1] found in their studies that survivors were significantly younger. Although our mean hospitalization time can be considered as long, this period includes the time taken for reconstructive surgery and convalescence. This correlates with the literature [6].

The etiology of FG was frequently polymicrobial and included aerobic, anaerobic and fungal organisms. In the literature, the most commonly identified microorganisms were E. coli, Bacteroides spp., staphylococci, Proteus spp., streptococci, Pseudomonas spp. and enterococci [2, $7,12-16]$. In our study, similar results were observed and no anaerobic microorganisms were isolated from the wound cultures. The etiologic factors identified in 68 of the patients were presumed to be the cause of the infectious processes. The microbial etiology was not significantly related to mortality ( $p>0.05$ ). In a recent study, no significant difference was shown between survivors and nonsurvivors in the type or number of isolated microorganisms [10].

In several studies, sepsis, diabetes, renal failure, congestive heart failure, coagulopathy, pulmonary embolism and pneumonia were mentioned as the major factors for mortality $[7,17]$. However, the relationship between mortality rate and number of predisposing factor has not been discussed widely in the literature. In an elegant study, Laor et al. [1], reported that the majority of his patients had at least one predisposing or concurrent medical or surgical illness, and among the 13 patients who died, 10 had at least three illnesses while 4 of 17 survivors had at least two illnesses. In our series, patients with three or more predisposing factors had the highest mortality rate and this indicates that multiple predisposing factors are the most important factor for mortality. In the literature, diabetes mellitus is the most common predisposing factor for FG and is frequently observed (39-64\%) $[3,5,7]$. Similarly, in our study, $50 \%$ of patients and $35 \%$ of nonsurvivors had diabetes mellitus. In a recent study, it was reported that alcoholism, coexistence of neoplasms and abdominal wall affection were also associated with higher mortality [18].

The most effective treatment for FG is aggressive and early surgical intervention. As previously reported, early surgical intervention (within 2 days of admission) significantly reduced FG-case fatality compared with delayed intervention (within 3-5 days of admission) [11]. The effect on mortality rates of the extent of body surface area involved in the necrotizing process was defined clearly. According to the literature, some authors have suggested that extension of the disease is not predictive of outcome [1, 6]. However, Chawla et al. [12] found a high mortality rate for patients who had undergone frequent operations for the great extent of the disease. In another study, Tuncel et al. [2] suggested that this was one of the most important risk factors for mortality in patients with FG. In our study, the mean extent of the involved body surface area was higher in nonsurvivors, but the difference was not statistically significant $(\mathrm{p}=0.17)$.

As previously reported, early diagnosis and intervention do not always depend on physicians as the patients usually only consult in advanced phases [18]. In our study, the time between the onset of the symptoms and the intervention can be considered as long. This can be related to the fact that most of the cases were admitted after one or more visits to other medical care facilities, as our hospital is a tertiary medical care center.

A colostomy should be performed in patients with FG involving the anorectal area with a high risk for fecal contamination. In this study, a colostomy was required for 23 patients, 5 of whom subsequently died. The mortality rate was not related to the colostomy, as previously reported $[5,16]$.

Laor et al. [1] created an FGSI by modifying the acute physiology and chronic health evaluation II severity score that describes the acuity of the disease. The FGSI contains nine parameters (temperature, heart and respiratory rates, levels of sodium, potassium, creatinine, bicarbonate, and hematocrit and white blood cell count). In our study, although FGSI score was greater in nonsurvivors, the difference was found to be not statistically sig- 
nificant and we concluded that the FGSI had no prognostic value. In a recent study, Lujan Marco et al. [3] also found that the FGSI score was greater in nonsurvivors than survivors, but the difference was not significant. Similarly, some studies have shown no relationship between high FGSI scores and mortality [2]. Conversely, a recent study by Corcoran et al. [4] revealed that FGSI scores were sensitive and specific for predicting mortality rate. Roghmann et al. [19] also suggested applying the age-adjusted Charlson Comorbidity Index (ACCI) and the recently introduced surgical Apgar Score (sAPGAR), in addition to FGSI, as they are more easily calculated, generally applicable and well validated.

We also evaluated serum albumin and ALP levels in 32 patients. Neither group was significantly different for the mean level of albumin or ALP. Unlike our study, Tuncel et al. [2] found significant differences between survivors and nonsurvivors in albumin and ALP levels. Similarly, Lin et al. [20] found that serum albumin levels between survivors and nonsurvivors at admission were significantly different.
Recently, HBO started to be used in order to support the management of FG [21]. Especially for synergistic infections, involving Clostridium spp., HBO is believed to increase the bactericidal action of neutrophils [22]. However, its efficiency is not proven by statistical evidence, but by numerous and empirical data obtained from frequent use [23]. Although we do not have experience in the use of $\mathrm{HBO}$ in FG, it can decrease the hospital stay since it reduces inflammation and promotes tissue regeneration.

In conclusion, FG is a serious, life-threatening infection and a surgical emergency. Despite the use of broadspectrum antibiotics and early aggressive surgical debridements, patients with FG still carry a high risk of mortality and morbidity. Multiple predisposing factors predict a poor prognosis and correlate highly with mortality. Early recognition of the disease, proper management of the predisposing factors and aggressive surgical debridement is the most essential interventions for stopping the rapidly progressing infectious process of FG and they can improve the clinical outcomes.

\section{References}

>1 Laor E, Palmer LS, Tolia BM, Reid RE, Winter HI: Outcome prediction in patients with Fournier's gangrene. J Urol 1995;154:89-92.

-2 Tuncel A, Aydin O, Tekdogan U, Nalcacioglu V, Capar Y, Atan A: Fournier's gangrene: three years of experience with 20 patients and validity of the Fournier's gangrene severity index score. Eur Urol 2006;50:838843.

$\checkmark 3$ Lujan Marco S, Budía A, Di Capua C, Broseta E, Jiménez CF: Evaluation of a severity score to predict the prognosis of Fournier's gangrene. Br J Urol 2010;106:373-376.

4 Corcoran AT, Smaldone MC, Gibbons EP, Walsh TJ, Davies BJ: Validation of the Fournier's gangrene severity index in a large contemporary series. J Urol 2008;180:944948.

$\checkmark 5$ Kuo CF, Wang WS, Lee CM, Liu CP, Tseng $\mathrm{HK}$ : Fournier's gangrene: ten-year experience in a medical center in northern Taiwan. J Microbiol Immunol Infec 2007;40:500506.

6 Martínez-Rodríguez R, Ponce de León J, Caparrós J, Villavicencio $\mathrm{H}$ : Fournier's gangrene: a monographic urology center experience with twenty patients. Urol Int 2009;83: 323-328.

7 Eke N: Fournier's gangrene: a review of 1,726 cases. Br J Surg 2000;87:718-728.

$>8$ Basoglu M, Gul O, Yildirgan I, Balik AA, Ozbey I, Oren D: Fournier's gangrene: review of fifteen cases. Am Surg 1997;63:1019-1021.
-9 Villanueva-Sáenz E, Martínez HernándezMagro P, Valdés Ovalle M, Montes Vega J, Alvarez-Tostado F JF: Experience in management of Fournier's gangrene. Tech Coloproctol 2002;6:5-13.

10 Altarac S, Katušin D, Crnica S, Papeš D, Rajković Z, Arslani N: Fournier's gangrene: etiology and outcome analysis of 41 patients. Urol Int 2012;88:289-293.

11 Sugihara T, Yasunaga $\mathrm{H}$, Horiguchi $\mathrm{H}, \mathrm{Fu}-$ jimura T, Ohe K, Matsuda S, Fushimi K, Homma Y: Impact of surgical intervention timing on the case fatality rate for Fournier's gangrene: an analysis of 379 cases. BJU Int DOI: 10.1111/j.1464-410X.2012.11291.x.

12 Chawla SN, Gallop C, Mydlo JH: Fournier's gangrene: an analysis of repeated surgical debridement. Eur Urol 2003;43:572-575.

13 Morpurgo E, Galandiuk S: Fournier's gangrene. Surg Clin North Am 2002;82:12131224

14 Laucks SS: Fournier's gangrene. Surg Clin North Am 1994;74:1339-1352.

15 Rotondo N: Fournier's gangrene: an unusual presentation of sepsis. J Emerg Med 2002;23: 413-414.

16 Yanar H, Taviloglu K, Ertekin C, Guloglu R, Zorba U, Cabioglu N, Baspinar I: Fournier's gangrene: risk factors and strategies for management. World J Surg 2006;30:1750 1754 .
17 Gürdal M, Yücebas E, Tekin A, Beysel M, Aslan R, Sengör F: Predisposing factors and treatment outcome in Fournier's gangrene. Analysis of 28 cases. Urol Int 2003;70:286290

18 Ruiz-Tovar J, Córdoba L, Devesa JM: Prognostic factors in Fournier gangrene. Asian J Surg 2012;35:37-41

19 Roghmann F, von Bodman C, Löppenberg B, Hinkel A, Palisaar J, Noldus J: Is there a need for the Fournier's gangrene severity index? Comparison of scoring systems for outcome prediction in patients with Fournier's gangrene. BJU Int DOI: 10.1111/j.1464-410X. 2012.11082.x


Lin WC, Chang HK, Hsu JM, Lo KY, Hsu $\mathrm{HH}$ : Is Fournier's gangrene severity index useful for predicting outcome of Fournier's gangrene? Urol Int 2005;75:119-122.

-21 Escobar SJ, Slade HJB, Hunt TK, Cianci P: Adjuvant hyperbaric oxygen $\left(\mathrm{HBO}_{2}\right)$ for necrotizing fasciitis reduces mortality and amputation rate. Undersea Hyperb Med Soc 2005;32:437-443.

22 Passavanti G: Can hyperbaric oxygen therapy (HOT) have a place in the treatment of some urological diseases? Urologia 2010;77: 257-262.

23 Morgan MS: Diagnosis and management of necrotising fasciitis: a multiparametric approach. J Hosp Infect 2010;75:249-257. 